\title{
Nonassociative geometry: Towards discrete structure of spacetime
}

\author{
Alexander I. Nesterov \\ Departament of Physics, C.U.C.E.I., Guadalajara University, Guadalajara, Jalisco, Mexico \\ and \\ L.V. Kirensky Institute of Physics, Siberian Branch Russian Academy of Science, Krasnoyarsk, Russia \\ E-mail: nesterov@udgserv.cencar.udg.mx; http://udgserv.cencar.udg.mx/ nesterov \\ Lev V. Sabinin \\ Department of Mathematics, Quintana Roo University, Chetumal, Mexico \\ and \\ Russian Friendship University, Moscow, Russia \\ E-mail: lsabinin@balam.cuc.uqroo.mx
}

\begin{abstract}
In the framework of nonassociative geometry a unified description of continuum and discrete spacetime is proposed. In our approach at the Planck scales the spacetime is described as a so-called diodular discrete structure which at large spacetime scales "looks like" a differentiable manifold. After a brief review of foundations of nonassociative geometry, we discuss the nonassociative smooth and discrete de Sitter spacetimes.
\end{abstract}

PACS numbers: 04.20.-q; 04.20.Gz; 02.40.Hw

Numerous attempts to construct the quantum theory of gravitation and to understand the structure of spacetime has not been successful so far and the problem is still open (for recent reviews see: [1] 5]). In general one should distinguish two strategies beyond the common treatment:

- Quantize a classical structure and then restore it as some kind of the classical limit of the quantum theory.

- Regard the classical structure as one being emerged from the other theory.

The second strategy may require a revision of the quantum theory itself in a way that that in quantum theory of gravitation the standard concept of spacetime must be replaced at the Planck scales by some kind of the discrete structure [1].

In this paper we propose in the framework of nonassociative geometry 6 10] a new approach to a classical and discrete structure of spacetime, which provides the unified description of continuum and discrete spacetime. The corresponding construction may be described as follows. In a neighborhood of an arbitrary point on a manifold with an affine connection one can introduce the geodesic local loop, which is uniquely defined by means of the parallel translation of geodesics along geodesics 122 14. The family of local loops constructed in this way uniquely defines the space with affine connection, but not every family of geodesic loops on a manifold defines an affine connection; there exist some relations between the loops at distinct points. Taking into account that the local loop sructure admits an additional operation, namely, the multiplication of the point by scalar, and a vector space structure induced by means of the exponential mapping; one can express the above mentioned relations by means of some algebraic identities. This leads to the notion of odule and the so-called geoodular structure.
A geoodular covering of the affinely connected manifolds, consisiting of the odular covering with some additional algebraic identities, contains complete information about the manifold and allows us to reconstruct it. If we take an arbitrary smooth geoodular covering, then it uniquely generates an affine connection, whose geoodular covering coincides with the initial one. Introducing the left invariant diodular metric, one obtains the Riemannian (pseudo-Riemannian) geodiodular manifold. This implies that a smooth geoodular (Riemannian/pseudo-Riemannian) manifold is an affinely connected (Riemannian/pseudo-Riemannian) manifold being described in another language and there is the equivalence of the corresponding categories [15, 16]. Ignoring the smoothness, it is possible to consider discrete spaces, introduce a diodular "metric" and a "connnection" over arbitrary fields or rings, and even to define finite spaces with "affine connection".

\section{Nonassociative geometry in brief}

Here we survey algebraic foundations of nonassociative geometry (for a recent review see: [9,10]).

Definition 1. Let $\langle Q, \cdot\rangle$ be a groupoid with a binary operation $(a, b) \mapsto a \cdot b$ and $Q$ be a smooth manifold. Then $\langle Q, \cdot\rangle$ is called a quasigroup if each of the equations $a \cdot x=$ $b, y \cdot a=b$ has a unique solution: $x=a \backslash b, y=b / a$. A loop is a quasigroup with a two-sided identity, $a \cdot e=$ $e \cdot a=a, \forall a \in Q$. A loop $\langle Q, \cdot, e\rangle$ with a smooth functions $\phi(a, b):=a \cdot b$ is called a smooth loop.

Let $\langle Q, \cdot, e\rangle$ be a smooth local loop with a neutral element $e$. We define

$$
L_{a} b=R_{b} a=a \cdot b, \quad l_{(a, b)}=L_{a \cdot b}^{-1} \circ L_{a} \circ L_{b},
$$


where $L_{a}$ is a left translation, $R_{b}$ is a right translation, $l_{(a, b)}$ is an associator.

Definition 2. Let $\langle M, \cdot, e\rangle$ be a partial groupoid with a binary operation $(x, y) \mapsto x \cdot y$ and a neutral element $e, x \cdot e=e \cdot x=x ; M$ be a smooth manifold (at least $C^{1}$-smooth) and the operation of multiplication (at least $C^{1}$-smooth) be defined in some neighborhood $U_{e}$. Then $\langle M, \cdot, e\rangle$ is called a partial loop on $M$.

Definition 3. Let $\langle M, \cdot, e\rangle$ be a left loop with a neutral element $e$ and $t: x \mapsto t x$ be a unary operation such that $(t+u) x=t x \cdot u x,(t u) x=t(u x)(t, u \in \mathbb{R}, x \in M)$. Then $\left\langle M, \cdot, e,(t)_{t \in \mathbb{R}}\right\rangle$ is called a left odule.

Definition 4. Let $M$ be a smooth manifold and

$$
L:(x, a, y) \in M \times M \times M \mapsto x_{a} y=L_{x}^{a} y \in M
$$

a smooth partial ternary operation, such that $L_{x}^{a} y$ defines in the some neighbourhood of the point $a$ the loop with the neutral $a$. Then the family $\left\{\left\langle M,{ }_{a}\right\rangle\right\}_{a \in M}$ is called a loopuscular structure.

A smooth manifold $M$ with a smooth partial ternary operation $L$ and smooth binary operations $t:(a, b) \in$ $M \times M \mapsto t_{a} b \in M, \quad(t \in \mathbb{R})$, such that $L_{x}^{a} y$ and $t_{a} x$ determine in some neighborhood of an arbitrary point $a$ the odule with the neutral element $a$, is called a left odular structure $\left\{\left\langle M, \dot{a}_{a},\left(t_{a}\right)_{t \in \mathbb{R}}\right\rangle\right\}_{a \in M}$. If $\left\{\left\langle M,{ }_{a},\left(t_{a}\right)_{t \in \mathbb{R}}\right\rangle\right\}_{a \in M}$ and $\left\{\left\langle M,{ }_{a}^{+},\left(t_{a}\right)_{t \in \mathbb{R}}\right\rangle\right\}_{a \in M}$ are odular structures, then $\left\{\left\langle M,{ }_{\dot{a}}, \stackrel{+}{a}^{+},\left(t_{a}\right)_{t \in \mathbb{R}}\right\rangle\right\}_{a \in M}$ is called a diodular structure. If $x_{a}^{+} y$ and $t_{a} x$ define a vector space, then such a diodular structure is called a linear diodular structure.

A diodular structure is said to be geodiodular if

(the first geoodular identity)

$L_{u_{a} x}^{t_{a} x} \circ L_{t_{a} x}^{a}=L_{u_{a} x}^{a}$

(the second geoodular identity)

(the third geoodular identity) $\quad L_{x}^{a}\left(y_{a} z\right)=L_{x}^{a} y_{a} L_{x}^{a} z$

are true.

The equivalence of the categories of geoodular (geodiodular) structures and of affine connections has been shown in 15, 16]. For a given loopuscular structure the tangent affine connection is defined by

$$
\begin{aligned}
& \nabla_{X_{a}} Y=\left\{\frac{d}{d t}\left(\left[\left(L_{g(t)}^{a}\right)_{*, a}\right]^{-1} Y_{g(t)}\right)\right\}_{t=0}, \\
& g(0)=a, \quad \dot{g}(0)=X_{a},
\end{aligned}
$$

$Y$ being a vector field in the neighborhood of a point $a$. In coordinates the components of this affine connection are

$$
\Gamma_{j k}^{i}(a)=-\left[\frac{\partial^{2}\left(L_{x}^{a} y\right)^{i}}{\partial x^{j} \partial y^{k}}\right]_{x=y=a}
$$

Definition 5. If $\{\langle M, \underset{a}{\dot{a}}\rangle\}_{a \in M}$ is a loopuscular structure then

$$
h_{(b, c)}^{a}=\left(L_{c}^{a}\right)^{-1} \circ L_{c}^{b} \circ L_{b}^{a}
$$

is called the elementary holonomy.

The elementary holonomy is, in fact, some integral curvature. In the smooth case, differentiating $\left(h_{(x, y)}^{a} z\right)^{i}$ by $x^{l}, y^{k}, z^{j}$ at $a \in M$ we get the curvature tensor at $a \in M$ precisely up to a numerical factor,

$$
R_{j k l}^{i}(a)=2\left[\frac{\partial^{3}\left(h_{(x, y)}^{a} z\right)^{i}}{\partial x^{l} \partial y^{k} \partial z^{j}}\right]_{x=y=z=a}
$$

Comment. Having the elementary holonomy $h_{(x, y)}$ at a neutral element, one can obtain the diodular structure in a unique way [9]:

$$
\begin{aligned}
& L_{x}^{a} y=L_{x}^{e} h_{(a, x)}\left(L_{a}^{e}\right)^{-1} y, \\
& x_{+}^{+} y=L_{a}^{e}\left(\left(L_{a}^{e}\right)^{-1} x_{e}^{+}\left(L_{a}^{e}\right)^{-1} y\right), \\
& t_{a} y=L_{a}^{e} t_{e}\left(L_{a}^{e}\right)^{-1} y .
\end{aligned}
$$

One may define left invariant metric $g^{a}(x, y)$ satisfying

$$
g^{b}\left(L_{b}^{a} x, L_{b}^{a} y\right)=g^{a}(x, y)
$$

In this way we obtain a metric diodular structure.

An odule $\left\langle M,{ }_{a}^{+},\left(t_{a}\right)_{t \in \mathbb{R}}\right\rangle$ replaces the tangent space at a point $a$ and we call it an osculating space. An element of the osculating space is called an osculating vector and in a smooth case it is a geodesic arc starting at the point $a$. An osculating structure $\left\{\left\langle M, \underset{a}{+},\left(t_{a}\right)_{t \in \mathbb{R}}\right\rangle\right\}_{a \in M}$ plays the role of tangent bundle structure of the smooth manifold. In the table below we compare the basic concepts of the classical differential geometry and of the nonassociative geometry.

\section{Differential Geometry Vs. Nonassociative Geometry}

\begin{tabular}{|l|l|}
\hline \hline Differential Geometry & Non-associative Geometry \\
\hline \hline Tangent space & Osculating space \\
\hline Tangent bundle structure & Osculating structure \\
\hline Cotangent space & Co-osculating space \\
\hline Parallel displacement & Left translations $L_{x}^{a} y$ \\
\hline Curvature $R(X, Y) Z$ & Elementary holonomy $h_{(x, y)}^{a} z$ \\
\hline Bianchi identities & Odular Bianchi identities \\
\hline \hline
\end{tabular}

Nonassociative discrete space. Here we consider a geodiodular discrete space $\mathcal{M}=\left\{\left\langle M, \underset{a}{\dot{a},{ }_{a}^{+}},\left(t_{a}\right)_{t \in \mathbb{R}}\right\rangle\right\}_{a \in M}$. This implies that there exists geodiodular covering of $M$ and:

1. $\left\langle M,_{\dot{a}},\left(t_{a}\right)_{t \in \mathbb{R}}\right\rangle$ is an odule with a neutral $a \in M$, for any $a \stackrel{a}{\in} M$, 
2. $\left\langle M, \underset{a}{+},\left(t_{a}\right)_{t \in \mathbb{R}}\right\rangle$ is a n-dimensional vector space (with zero element $a \in M$ ),

3. The following geoodular identities

(the first geoodular identity) $\quad L_{u_{a} x}^{t_{a} x} \circ L_{t_{a} x}^{a}=L_{u_{a} x}^{a}$,

(the second geoodular identity) $L_{x}^{a} \circ t_{a}=t_{x} \circ L_{x}^{a}$,

(the third geoodular identity) $\quad L_{x}^{a}\left(y_{a} z\right)=L_{x}^{a} y_{x} L_{x}^{a} z$

are valid. One may consider the operations above as partially defined.

In our approach the osculating space $\mathcal{M}_{a}^{+}=$ $\left\langle M,_{a}^{+}, a,\left(t_{a}\right)_{t \in \mathbb{R}}\right.$ plays the role of tangent space at $a \in M$. Indeed, in the smooth case the tangent space $T_{a} M$ may be identified, at least locally, with $M$ by means of the exponential map. Any point $x \in M$ may be, then, regarded as an osculating vector in $\mathcal{M}_{a}^{+}(\forall a \in M)$. Any line $\left(t_{a} b\right)_{t \in \mathbb{R}}$ may be regarded as a geodesic through $a, b \in M$.

The presence of curvature in such a space results in a non-trivial elementary holonomy,

$$
L_{a}^{y} \circ L_{y}^{x} \circ L_{x}^{a}=h^{a}(x, y) \neq \mathrm{Id} .
$$

Having given a discrete nonassociative space (finite affinely connected space in other words) we can enrich it by a metric diodular structure. Namely, one may define additionally non-degenerate left invarint metric $g^{a}(x, y)$ for any $\mathcal{M}_{a}^{+}: g^{b}\left(L_{b}^{a} x, L_{b}^{a} y\right)=g^{a}(x, y)$.

\section{Nonassociative de Sitter spacetime}

The spacetimes of constant curvature are locally characterized by the condition

$$
R_{\mu \nu \lambda \sigma}=K\left(g_{\mu \lambda} g_{\nu \sigma}-g_{\mu \sigma} g_{\nu \lambda}\right) .
$$

One can regard these spaces as solutions of the Einstein's equations with $\Lambda$-term for an empty space and $\Lambda=3 K$.

The spacetime of constant curvature with $K>0$ is called de Sitter spacetime, and if $K<0$ it is called antide Sitter spacetime. De Sitter spacetime has the topology $S^{3} \times \mathbb{R}$ and can be considered as hyperboloid [17.18

$$
Z_{a} Z^{a}-Z_{0} Z^{0}=-1 / K, a=1, \ldots, 4
$$

embedded into five-dimensional Minkowski space with metric

$$
d s^{2}=\left(d Z^{0}\right)^{2}-d Z_{a} d Z^{a}
$$

In the remainder of this section we consider de Sitter spacetime with the inner metric 18

$$
d s^{2}=\frac{d t^{2}-d x^{2}-d y^{2}-d z^{2}}{\left(1-\frac{K}{4}\left(t^{2}-x^{2}-y^{2}-z^{2}\right)\right)^{2}},
$$

but our approach may be extended to anti-de Sitter spacetime as well.
Smooth de Sitter spacetime. The easiest way to study the spacetimes of the constant curvature in the framework of nonassociative geometry is to employ the quaternions. Let us consider the quaternionic algebra over complex field $\mathbb{C}(1, i)$

$$
\mathrm{H}_{\mathcal{C}}=\{\alpha+\beta i+\gamma j+\delta k \mid \alpha, \beta, \gamma, \delta \in \mathbb{C}\}
$$

with multiplication operation defined by the property of bilinearity and following rules for $i, j, k$ :

$$
\begin{aligned}
& i^{2}=j^{2}=k^{2}=-1, \quad j k=-k j=i, \\
& k i=-i k=j, \quad i j=-j i=k .
\end{aligned}
$$

The quaternionic conjugation (denoted by ${ }^{+}$) is defined by

$$
q^{+}=\alpha-\beta i-\gamma j-\delta k
$$

for $q=\alpha+\beta i+\gamma j+\delta k$. This definition implies

$$
(q p)^{+}=p^{+} q^{+}, \quad p, q \in \mathrm{H}_{\mathcal{C}} .
$$

Further we restrict ourselves to the set of quaternions $\mathrm{H}_{\mathbb{R}}$ :

$$
\begin{aligned}
\mathrm{H}_{\mathbb{R}}= & \left\{\zeta=\zeta^{0}+\mathrm{i}\left(\zeta^{1} i+\zeta^{2} j+\zeta^{3} k\right): \mathrm{i}^{2}=-1, \mathrm{i} \in \mathbb{C},\right. \\
& \left.\zeta^{0}, \zeta^{1}, \zeta^{2}, \zeta^{3} \in \mathbb{R}\right\}
\end{aligned}
$$

with the norm $\|\zeta\|^{2}$ given by

$$
\|\zeta\|^{2}=\zeta \zeta^{+}=\left(\zeta^{0}\right)^{2}-\left(\zeta^{1}\right)^{2}-\left(\zeta^{3}\right)^{2}-\left(\zeta^{4}\right)^{2} .
$$

Introducing a binary operation

$$
\zeta * \eta=(\zeta+\eta) /\left(1+\frac{K}{4} \zeta^{+} \eta\right), \zeta, \eta \in \mathrm{H}_{\mathbb{R}}
$$

where $K$ is constant and / denotes the right division, we find that the set of quaternions $\mathrm{H}_{\mathbb{R}}$ with the binary operation $*$ forms a loop $Q \mathrm{H}_{\mathbb{R}}$ which admits a natural geodiodular structure induced by the quaternionic algebra. The associator is found to be

$$
l_{(\zeta, \eta)} \xi=\left(1+\frac{K}{4} \zeta \eta^{+}\right) \xi /\left(1+\frac{K}{4} \zeta^{+} \eta\right) .
$$

Employing (8), we define the left invariant diodular metric on $Q \mathrm{H}_{\mathbb{R}}$ as follows:

$$
\begin{aligned}
g^{\zeta}(\xi, \eta)= & \frac{(\xi-\zeta)\left(1-\frac{K}{4} \xi^{+} \zeta\right)\left(1-\frac{K}{4} \zeta^{+} \eta\right)\left(\eta^{+}-\zeta^{+}\right)}{2\left\|1-\frac{K}{4} \zeta^{+} \xi\right\|^{2}\left\|1-\frac{K}{4} \zeta^{+} \eta\right\|^{2}}+ \\
& +\frac{(\eta-\zeta)\left(1-\frac{K}{4} \eta^{+} \zeta\right)\left(1-\frac{K}{4} \zeta^{+} \xi\right)\left(\xi^{+}-\zeta^{+}\right)}{2\left\|1-\frac{K}{4} \zeta^{+} \xi\right\|^{2}\left\|1-\frac{K}{4} \zeta^{+} \eta\right\|^{2}}
\end{aligned}
$$

In particular,

$$
g^{0}(\xi, \eta)=\frac{1}{2}\left(\xi \eta^{+}+\eta \xi^{+}\right)=\xi^{\nu} \eta_{\nu}
$$

and 


$$
g^{\zeta}(\xi, \xi)=\frac{\|\xi-\zeta\|^{2}}{\left\|1-\frac{K}{4} \zeta^{+} \xi\right\|^{2}} .
$$

Let $\xi=\zeta+d \zeta$, then (17) leads to the de Sitter metric (10):

$$
g(d \zeta, d \zeta)=\frac{\left(d \zeta^{0}\right)^{2}-\left(d \zeta^{1}\right)^{2}-\left(d \zeta^{2}\right)^{2}-\left(d \zeta^{3}\right)^{2}}{\left(1-\frac{K}{4}\left(\left(\zeta^{0}\right)^{2}-\left(\zeta^{1}\right)^{2}-\left(\zeta^{2}\right)^{2}-\left(\zeta^{3}\right)^{2}\right)\right)^{2}} .
$$

Taking into account that for symmetric spaces its elementary holonomy is determined by the associator [6,9:

$$
h_{(\zeta, \eta)} \xi=l_{\left(\zeta, L_{\zeta}^{-1} \eta\right)} \xi,
$$

we find

$$
h_{(\zeta, \eta)} \xi=\left(1-\frac{K}{4} \eta \zeta^{+}\right) \xi /\left(1-\frac{K}{4} \eta^{+} \zeta\right) .
$$

Applying (14), we obtain the curvature tensor of de Sitter spacetime in the normal coordinates as the following:

$$
R_{\mu \nu \lambda \sigma}=-\frac{K}{2} \varepsilon_{\mu \nu \kappa \delta} \varepsilon_{\lambda \sigma}^{\kappa \delta} .
$$

Comment. The de Sitter spacetime may be obtained as a solution of the diodular Einstein's equations with ' $\Lambda$-term' 10,11.

Discrete de Sitter spacetime. Let us consider a finite set $M=\mathbb{Z}_{n}^{4}=\left\{\mathbf{p}=\left(p^{\mu}\right) \mid p^{\mu} \in \mathbb{Z}_{n}, \mu=0, \ldots, 3, n \in \mathbb{N}\right\}$ where $\mathbb{Z}_{n}=\{p=-n, \ldots, n\}$ is the set of integers. We define a partial loop $Q \mathrm{H}_{\mathbb{Z}_{n}^{4}}$ as a set of quaternions

$$
\begin{aligned}
\mathrm{H}_{\mathbb{Z}_{n}^{4}}= & \left\{\zeta_{\mathbf{p}}=\ell\left(p^{0}+\mathrm{i}\left(p^{1} i+p^{2} j+p^{3} k\right)\right): \ell=\mathrm{const},\right. \\
& \left.\mathrm{i}^{2}=-1, \mathrm{i} \in \mathbb{C} ; \mathbf{p} \in \mathbb{Z}_{\mathrm{n}}^{4}\right\}
\end{aligned}
$$

with the indefinite norm $\left\|\zeta_{\mathbf{p}}\right\|^{2}=\ell^{2} p^{\mu} p_{\mu}$ and the binary operation $\mathrm{H}_{\mathbb{Z}_{n}^{4}} \times \mathrm{H}_{\mathbb{Z}_{n}^{4}} \mapsto \mathrm{H}_{\mathbb{Z}_{n}^{4}}$ defined by

$\zeta_{\mathbf{p}} * \zeta_{\mathbf{q}}=\zeta_{\mathbf{p q}}=\left(\zeta_{\mathbf{p}}+\zeta_{\mathbf{q}}\right) /\left(1+\frac{K}{4} \zeta_{\mathbf{p}}^{+} \zeta_{\mathbf{q}}\right), \quad \zeta_{\mathbf{p}}, \zeta_{\mathbf{q}} \in \mathrm{H}_{\mathbb{Z}_{n}^{4}}$.

We introduce a partial geodiodular finite space $\mathcal{M}_{n}$ at the neutral element $e$ (zero) as follows:

- $Q \mathrm{H}_{\mathbb{Z}_{n}^{4}}$ being the odule with the unary operation multiplication induced by the quaternionic algebra over $\mathbb{Z}$

- $\mathcal{M}^{+}=\mathrm{H}_{\mathbb{Z}_{n}^{4}}$ being the osculating space with the structure of vector space induced by the quaternionic algebra over $\mathbb{Z}$.

Employing the geoodular identities one can obtain the geoodular covering of the discrete spacetime.

For the duiodular metric and elementary holonomy we have

$$
\begin{aligned}
& g^{\zeta_{\mathbf{p}}}\left(\zeta_{\mathbf{q}}, \zeta_{\mathbf{q}}\right)=\frac{\left\|\zeta_{\mathbf{p}}-\zeta_{\mathbf{q}}\right\|^{2}}{\left\|1-\frac{K}{4} \zeta_{\mathbf{p}}^{+} \zeta_{\mathbf{q}}\right\|^{2}}, \\
& h_{\left(\zeta_{\mathbf{p}}, \zeta_{\mathbf{q}}\right)} \zeta_{\mathbf{m}}=\left(1-\frac{K}{4} \zeta_{\mathbf{q}} \zeta_{\mathbf{p}}^{+}\right) \zeta_{\mathbf{m}} /\left(1-\frac{K}{4} \zeta_{\mathbf{q}}^{+} \zeta_{\mathbf{p}}\right) \text {. }
\end{aligned}
$$

The smooth spacetime could be regarded as the result of "limit process of triangulating" when $\ell n=$ const, while $\ell \longrightarrow 0, n \longrightarrow \infty$. Let us consider $\mathbf{q}=\mathbf{p}+\boldsymbol{\delta},|\boldsymbol{\delta}| \ll n$. Then we have $\zeta_{\mathbf{q}}=\zeta_{\mathbf{p}}+\Delta \zeta_{\mathbf{q}}$ where $\Delta \zeta_{\mathbf{q}}=\ell\left(\delta^{0}+\mathrm{i}\left(\delta^{1} i+\right.\right.$ $\left.\delta^{2} j+\delta^{3} k\right)$ ), and the diodular metric (21) takes the form

$$
g^{\zeta_{\mathbf{p}}}\left(\zeta_{\mathbf{q}}, \zeta_{\mathbf{q}}\right)=\frac{\left\|\Delta \zeta_{\mathbf{q}}\right\|^{2}}{\left\|1-\frac{K}{4} \zeta_{\mathbf{p}}^{+} \zeta_{\mathbf{p}}\right\|^{2}}+O\left(K \ell^{2}\right)
$$

and

$g^{\zeta_{\mathbf{p}}}\left(\zeta_{\mathbf{q}}, \zeta_{\mathbf{q}}\right) \longrightarrow d s^{2}=\frac{\left(d \zeta^{0}\right)^{2}-\left(d \zeta^{1}\right)^{2}-\left(d \zeta^{2}\right)^{2}-\left(d \zeta^{3}\right)^{2}}{\left(1-\frac{K}{4}\left(\left(\zeta^{0}\right)^{2}-\left(\zeta^{1}\right)^{2}-\left(\zeta^{2}\right)^{2}-\left(\zeta^{3}\right)^{2}\right)^{2}\right.}$,

while $\ell \rightarrow 0, n \rightarrow 0$. Comparing with the smooth case, we see that to some extent the information concerning the geometry of de Sitter spacetime is hidden in the structure of finite loop.

The similar consideration of the elementary holonomy gives

$$
h_{\left(\zeta_{\mathbf{p}}, \zeta_{\mathbf{q}}\right)} \zeta_{\mathbf{m}}=\zeta_{\mathbf{m}}+\frac{K}{4} \Delta\left(\zeta_{\mathbf{p}}, \zeta_{\mathbf{q}}, \zeta_{\mathbf{m}}\right)+O\left(K \ell^{2}\right),
$$

where

$$
\Delta\left(\zeta_{\mathbf{p}}, \zeta_{\mathbf{q}}, \zeta_{\mathbf{m}}\right)=\zeta_{\mathbf{m}} \zeta_{\mathbf{q}}^{+} \zeta_{\mathbf{p}}-\zeta_{\mathbf{q}} \zeta_{\mathbf{p}}^{+} \zeta_{\mathbf{m}}
$$

In the coordinates (24) can be written as

$$
\left(h_{\left(\zeta_{\mathbf{p}}, \zeta_{\mathbf{q}}\right)}\right)_{\nu}^{\mu} \zeta_{\mathbf{m}}^{\nu}=\zeta_{\mathbf{m}}^{\mu}+\frac{K}{4} \varepsilon_{\mu \nu \kappa \delta} \varepsilon_{\lambda \sigma}^{\kappa \delta} \zeta_{\mathbf{m}}^{\nu} \zeta_{\mathbf{p}}^{\lambda} \zeta_{\mathbf{q}}^{\sigma}+O\left(K \ell^{2}\right)
$$

Approaching the limit, while $n \longrightarrow \infty, \ell \longrightarrow 0$, one restores the curvature tensor of de Sitter spacetime in the normal coordinates:

$$
R_{\mu \nu \lambda \sigma}=-\frac{K}{2} \varepsilon_{\mu \nu \kappa \delta} \varepsilon_{\lambda \sigma}^{\kappa \delta} .
$$

The above examples show how the continuum and discrete structure of spacetime might be described in the framework of the nonassociative geometry. We deal with points and essentially nonassociative operation. This leads us to the concept of the nonassociative (discrete) spacetime, when at distances comparables with Planck length the standard concept of spacetime might be replaced by the diodular discrete structure which at large spacetime scales "looks like" a differentiable manifold.

[1] J. Butterfield and C.J.Isham, Spacetime and the Philosophical Challenge of Quantum Gravity, gr-qc/9903072. 
[2] C.J. Isham, Structural Issues in Quantum Gravity, grqc/9510063.

[3] C. Rovelli, Quantum spacetime: what do we know? grqc/9903045

[4] C. Rovelli, Loop Quantum Gravity.Living Reviews in Relativity (refereed electronic journal), http://www.livingreviews.org/Articles/Volume1/19981rovelli; gr-qc/9709008.

[5] C. Rovelli, String, loops and others: a critical survey of the presence approaches to quantum gravity, $\mathrm{gr}-$ qc/9803024.

[6] L.V. Sabinin, Differential equations of smooth loops, in: Proc. of Sem. on Vector and Tensor Analysis, 23133. Moscow: Moscow Univ. 1988.

[7] L.V. Sabinin, Differential Geometry and Quasigroups, Proc. Inst. Math. Siberian Branch of Ac. Sci. USSR, 14, 208 (1989).

[8] L.V. Sabinin, On differential equations of smooth loops, Russian Mathematical Survey, 49172 (1994).

[9] L.V. Sabinin, Smooth quasigroups and loops, Dordrecht: Kluwer Academic Publishers (1999).

[10] A.I. Nesterov and L.V. Sabinin, Non-aasociative geometry and discrete structure of spacetime, Comment. Math. Univ. Carolinae 41,2, 347 (2000), hep-th/0003238.

[11] A.I. Nesterov, and L.V. Sabinin, in preparation.

[12] M. Kikkawa, On local loops in affine manifolds, J. Sci. Hiroshima Univ. Ser A-I Math. 28, 199 (1961).

[13] L.V. Sabinin, The geometry of loops, Mathematical Notes, 12, 799 (1972).

[14] L.V. Sabinin, On the equivalence of categories of loops and homogeneous spaces, Soviet Math. Dokl. 13, 970 (1972).

[15] L.V. Sabinin, Odules as a new approach to a geometry with a connection, Soviet Math. Dokl. 18, 515 (1977).

[16] L.V. Sabinin, Methods of Nonassociative Algebra in Differential Geometry, in Supplement to Russian translation of S.K. Kobayashi and K. Nomizy "Foundations of Differential Geometry", Vol. 1. Moscow: Nauka (1981).

[17] S.W. Hawking and G.F.R. Ellis, The large scale structure of space-time. Cambridge: Univ. Press. (1975)

[18] D. Kramer, H. Stephani, M. MacCallum and E. Herlt,Exact Solutions of Einstein's Equations, Berlin: VEB Deutscher Verlag (1980). 